\title{
Seasonality of hypoplastic left heart syndrome in the United States: A 10-year time-series analysis
}

\author{
Pirooz Eghtesady, MD, PhD, ${ }^{\mathrm{a}, \mathrm{b}}$ Anoop Brar, $\mathrm{PhD},{ }^{\mathrm{b}}$ and Matthew Hall, $\mathrm{PhD}^{\mathrm{c}}$
}

\begin{abstract}
Objective: Hypoplastic left heart syndrome is a major congenital heart defect and is associated with significant morbidity and mortality. Its etiology remains unknown although genetic studies imply complex inheritance. Anecdotal reports of cluster presentations suggest the possible involvement of an environmental component, although previous epidemiologic studies have been of limited scope. The objective of this study was to examine seasonal and temporal patterns of hypoplastic left heart syndrome births compared with other left-sided heart defects in the United States.
\end{abstract}

\begin{abstract}
Methods: We conducted a retrospective analysis of the Pediatric Health Information System inpatient database from pediatric hospitals across the country from 1996 to 2006. Population and index case patterns were analyzed for each diagnostic category. An epidemiologic survey was performed through time-series analyses using Fisher's Kappa test and the Bartlett Kolmogorov-Smirnov test. The existence and strength of seasonality for the left-sided heart defects was quantified by the autoregression $R^{2}$.
\end{abstract}

Results: A seasonal occurrence was found in hypoplastic left heart syndrome but not other left-sided heart diseases. Significant seasonal differences occurred each year, with peaks in summer months and troughs in winter months. The seasonality inversely correlated with the incidence of chromosomal and extracardiac anomalies; such anomalies were highest in interrupted aortic arch, which had a random pattern of presentation.

Conclusions: There is a significant seasonal pattern in the presentation of hypoplastic left heart syndrome, with preponderance in summer months, in contrast to the random pattern in other left-sided heart diseases. Further studies are warranted to identify the influence of potential environmental factor(s) in hypoplastic left heart syndrome, as seen in diseases with seasonal patterns. (J Thorac Cardiovasc Surg 2011;141:432-8)

\begin{abstract}
"They come in spurts, don't they?", is a comment often heard in response to news of another admission of an infant with hypoplastic left heart syndrome (HLHS) to the intensive care unit. This statement reflects the sense that affected infants and those with related defects (eg, Shone anomaly) frequently appear to present in clusters. Such anecdotal experience, however, has not previously been studied objectively. The etiology of HLHS and related diseases, collectively herein referred to as left-sided congenital heart defects, remains unknown. They account for only about $14 \%$ of congenital heart defects but represent a substantially greater burden of morbidity and mortality because of their effect on the primary structures involved in supporting the circulation. These defects comprise a spectrum of congenital cardiac anomalies with HLHS carrying the highest mortality, ${ }^{1}$ although outcomes are improving. ${ }^{2}$ A variety of causative factors has been implicated, including abnormal

From the Division of Cardiothoracic Surgery, ${ }^{\mathrm{a}}$ Cincinnati Children's Hospital, ${ }^{\mathrm{b}}$ Cincinnati, Ohio; and Child Health Corporation of America, ${ }^{\mathrm{c}}$ Shawnee Mission, Kan. Disclosures: Authors have nothing to disclose with regard to commercial support.

Received for publication Jan 21, 2010; revisions received April 8, 2010; accepted for publication June 29, 2010; available ahead of print Sept 3, 2010.

Address for reprints: Pirooz Eghtesady MD, PhD, Division of Cardiothoracic Surgery, Cincinnati Children's Medical Center, 3333 Burnet Ave, Cincinnati, Ohio 452293039 (E-mail: Pirooz.Eghtesady@cchmc.org).

$0022-5223 / \$ 36.00$

Copyright (c) 2011 by The American Association for Thoracic Surgery doi:10.1016/j.jtcvs.2010.06.060
\end{abstract}

intrauterine blood flow patterns, hereditary factors, intrauterine infection, and myocarditis or immunologic injury. ${ }^{3-6}$ Genetic studies ${ }^{7,8}$ show that the incidence of HLHS does not follow simple mendelian genetics but exhibits "complex inheritance" with contributions from both genetic and other undefined factors.

To better elucidate potential etiologic mechanisms, investigators have examined the influence of maternal factors, ${ }^{9,10}$ race, ${ }^{4,11}$ ethnicity, ${ }^{12}$ environmental exposure, ${ }^{10,11}$ and gender, ${ }^{4,11}$ on the epidemiology of HLHS and left-sided congenital heart diseases. Many of these studies were performed at a time when palliative reconstruction was not favorable, resulting in limited sample sizes ${ }^{9,13}$ Along with improvements in prenatal diagnosis, higher survivals after surgical palliation have resulted in greater referral of affected infants to major tertiary centers. The purpose of this study was to examine the epidemiologic distribution of left-sided congenital heart diseases in a multi-institutional database, with particular emphasis on HLHS.

\section{METHODS \\ Source of Data}

Patients with HLHS and 3 other left-sided congenital heart defects, defined below, were identified through the Pediatric Health Information System (PHIS), an administrative inpatient database developed by the Child Health Corporation of America (Shawnee Mission, Kan) from not-forprofit, freestanding pediatric hospitals across the United States. Data derived 


\section{Abbreviations and Acronyms \\ AS = aortic stenosis \\ CoA $=$ coarctation of the aorta \\ HLHS = hypoplastic left heart syndrome \\ IAA = interruption of the aortic arch \\ ICD-9 = International Classification of Disease- ninth revision \\ PHIS $=$ Pediatric Health Information System}

from 24 hospitals with complete data for every month of every year from 1996 to 2006 were used for analysis. For external benchmarking, participating hospitals provide quarterly data that undergo rigorous quality and reliability assessment. Standardized data on demographics, diagnoses, procedures, interventions, and outcomes for patients discharged were extracted. Incidence rates from the Centers for Disease Control National Vital Statistics (1996-2006) were used to obtain monthly live births. The Institutional Review Board at Cincinnati Children's Hospital Medical Center approved this study.

\section{Case Selection}

Inclusion criteria required subjects to have at least one International Classification of Disease-ninth revision (ICD-9) discharge diagnosis code (Table 1). Patients were classified into 1 of 4 diagnosis groups: coarctation of the aorta (CoA), congenital stenosis of the aortic valve (AS), HLHS, and interruption of the aortic arch (IAA). These patients had no other associated left-sided congenital heart diseases. All entries were audited, blinded to birth-date data to ensure that there was no miscoding for the neonates (eg, a CoA being identified as congenital AS on a subsequent admission). Inasmuch as patients may change institutions after diagnosis, patients older than 10 days of age at admission or less than 10 days at discharge were excluded to avoid double-counting of patients. ${ }^{14}$ Potentially, we may be underestimating the incidence of congenital AS or CoA, inasmuch as those patients with less severe disease, perhaps not requiring intervention in the neonatal period, are less likely to be treated at a tertiary care center compared with HLHS. These numbers would not, however, affect our analysis to determine the possibility of a seasonality pattern of presentation. To address the preceding and to assess for sensitivity analysis, we compared data from babies at 6 months or younger for CoA, congenital AS, and HLHS.

The incidence of multiple congenital anomalies, widely known to be associated with genetic aberrations, was extracted using ICD-9 codes (Table 1) not only to allow characterization of populations but also to enable determinations of differences across populations.

\section{Data Analysis}

Owing to their nonnormal distributions, continuous demographics are presented using medians and interquartile ranges (25th percentile, 75 th percentile). These demographics were compared across the 4 abnormality categories using the Wilcoxon rank sum tests. Categorical demographics are presented with frequencies and percents, and comparisons across the abnormalities are made with $\chi^{2}$ tests.

To test for seasonality, we used time-series analyses for the rates of each abnormality per 100,000 births. First, tests for stationarity (ie, an increasing or decreasing trend) of each series were performed using the Dickey-Fuller unit root test and differencing of series was performed when necessary. Second, to detect whether seasonality existed for each of the diagnostic categories, we used Fisher's Kappa and the Bartlett Kolmogorov-Smirnov tests. Although these tests indicate the presence of a seasonal trend, they do not provide a measure for the strength or magnitude of the seasonality, for which we determined the autoregressive coefficient of $R^{2}{ }_{\text {Autoreg. }}{ }^{15}$ The strength of the seasonality was classified as follows: (1) nonexistent or weak: $0 \leq R_{\text {Autoreg }}^{2}<0.4$, (2) moderate to strong: $0.4 \leq R_{\text {Autoreg }}^{2}<0.7$, or (3) strong to perfect: $0.7 \leq R_{\text {Autore }}^{2} \leq 1$. To estimate the magnitude of the seasonality, we found the difference in the maximum and minimum monthly estimates from the regression.

We also compared patients referred with HLHS in the months with the highest and lowest rates. To do this, we determined the 2 months during each year with the highest rates and the 2 with the lowest rates. We then compared demographic characteristics of patients in these 2 groups with Wilcoxon rank sum tests (continuous covariates) and $\chi^{2}$ (categorical covariates).

SAS version 9.1.3 (SAS Institute, Inc, Cary, NC) was used for all analyses with the \%DFTEST macro for the Dickey-Fuller test, PROC SPECTRA for Fisher's Kappa test and the Bartlett Kolmogorov-Smirnov test, and PROC AUTOREG for the strength and magnitude estimation. In the autoregression models, we used maximum likelihood estimates with backward elimination and a maximum order of 13 . Model-based monthly rate estimates were smoothed with a fitted penalized B-spline curve.

\section{RESULTS}

\section{Frequency Counts}

We identified 2763 patients from the PHIS database in 24 children's hospitals from across the United States spanning the decade of 1996 to 2006. Of the left-sided congenital heart diseases, the largest group had a primary diagnosis of HLHS $(n=1353$ or $49 \%)$, whereas CoA $(n=962$ or $34.8 \%)$, IAA $(\mathrm{n}=260$ or $9.4 \%)$, and congenital AS $(\mathrm{n}=188$ or $6.8 \%)$ were comparatively less prevalent.

\section{Demographic Characteristics on First Admission}

A greater prevalence of left-sided congenital heart diseases was seen in boys $(P=.011)$ (Table 2$)$; the highest disparity between genders was noted for congenital AS $(64.89 \% ; 95 \%$ confidence interval of $58.07-71.72)$ and HLHS $(62.08 \%$; $95 \%$ confidence interval of 59.50 64.67), and the lowest was for IAA $(52.69 \%$; $95 \%$ confidence interval of 46.62-58.76). Neonatal mortality during first hospital admission was highest in patients with HLHS (23.73\% of total), followed by congenital AS (11.17\%) and IAA $(11.15 \%)$, and lowest in those with CoA $(3.12 \%)$. Prevalence of the lesions did not vary by race (Table 2). The mean age at admission was 0 to 1 days for all groups except CoA, with HLHS having the most $(66.7 \%)$ admissions at this age. Approximately equal numbers of CoA patients were admitted between 0 and 1 day $(30.3 \%)$ and at more than 6 days $(37.3 \%)$. The birth weight of the majority $(80 \%-90 \%)$ of patients in all groups was more than $2500 \mathrm{~g}$, with only $10 \%$ to $20 \%$ weighing 1500 to $2499 \mathrm{~g}$. Gestational age did not differ across the groups $(P=.904)$, but age in days at admission $(P<.0001)$ and birth weight $(P=.040)$ did. HLHS patients tended to be younger, and IAA patients tended to have lower birth weights.

\section{Frequency of Patient Population With Specific Secondary Diagnoses}

Ventricular septal defect was the major secondary diagnosis in the majority $(88.1 \%$ ) of patients with IAA (Table 3 ). In 
TABLE 1. ICD-9 diagnosis codes for patient classification

\begin{tabular}{ll}
\hline ICD-9 diagnosis codes & \multicolumn{1}{c}{ Diagnosis } \\
\hline 747.10 & Coarctation of aorta \\
746.3 & Congenital stenosis of aortic valve \\
746.7 & Hypoplastic left heart syndrome \\
747.11 & Interruption of aortic arch \\
746.81 & Subaortic stenosis \\
746.5 & Congenital mitral stenosis \\
745.11 & Double-outlet right ventricle \\
745.10 & Transposition of the great arteries \\
745.69 & Endocardial cushion defects (complete \\
& atrioventricular canal, both balanced and \\
745.4 & nonbalanced) \\
746.81 & Ventricular septal defect \\
747.41 & Subaortic stenosis \\
747.42 & Total anomalous pulmonary venous return \\
753 & Partial anomalous venous return \\
742 & Kidney anomalies \\
$758.0-758.9$ & Nervous system anomalies \\
$748-759$ & Chromosomal anomalies including gonadal \\
\hline
\end{tabular}

Patient data were extracted based on the International Classification of Disease-ninth revision (ICD-9) diagnosis codes shown.

contrast, this defect occurred in only approximately half $(43.9 \%$ ) the patients with CoA, in only $23.9 \%$ with congenital AS, and in $11.5 \%$ with HLHS. Further, in our sample of subjects, those with congenital lesions such as double-outlet right ventricle or looping defects accounted for a very small fraction $(0.7 \%-3 \%)$ of all patients. Multiple congenital anomalies, particularly if 3 or more, are frequently attributable to genetic aberrations and are also associated with chromosomal defects. ${ }^{16}$ Further analysis of the data, therefore, was undertaken to determine the potential contribution of genetic and chromosomal aberrations to left-sided congenital heart diseases (Table 3). Chromosomal anomalies (other than Turner syndrome) in patients with IAA $(11.9 \%)$ were significantly $(P<.0001)$ more prevalent than in patients with CoA $(7.9 \%)$ or HLHS $(2.1 \%)$, based on a pairwise comparison. In patients with CoA, Turner syndrome and associated gonadal dysgenesis complexes accounted for nearly all the chromosomal anomalies and congenital anomalies.

We performed sensitivity analyses to determine whether the seasonality in the rates was related to methodologic decisions we made. First, we removed all patients with one of the secondary diagnoses listed in Table 3. Significance on the statistical tests for seasonal trends remained consistent with those displayed in Table 4. Additionally, we performed a second sensitivity analysis by removing the criteria that patients had to be 10 days of age or younger at admission and more than 10 days at discharge. Alternatively, we restricted our population to be less than 6 months of age at admission. Although rates of the diagnoses increased, the significance in the seasonal trends did not (data not shown).

\section{Seasonal Trends in Birth Month}

Spectral analysis was performed to detect seasonality by determining periodicity over time for the entire cohort. According to the Dickey-Fuller test, the HLHS group was the only nonstationary (ie, increasing) series $(P=.025)$ and required differencing. Overall, the upward trend in overall rates for left-sided congenital heart diseases could be accounted for by increased occurrence or detection of HLHS and CoA over the 10-year period analyzed.

The white noise test showed a strong periodicity or seasonality (Fisher's Kappa 15.86; $P<.001$; and the Bartlett Kolmogorov-Smirnov value of $0.30 ; P<.001$ ) for HLHS.

TABLE 2. Demographic characteristics of subjects on first admission

\begin{tabular}{|c|c|c|c|c|c|}
\hline & $\operatorname{CoA}(n=962)$ & Congenital AS $(n=188)$ & HLHS $(n=1353)$ & IAA $(n=260)$ & $P$ \\
\hline \multicolumn{6}{|l|}{ Sex } \\
\hline Female & $378(39.39)$ & $66(35.11)$ & $513(37.92)$ & $123(47.31)$ & .011 \\
\hline Male & $584(60.71)$ & $122(64.89)$ & $840(62.08)$ & $137(52.69)$ & \\
\hline \multicolumn{6}{|l|}{ Disposition } \\
\hline Died & $30(3.12)$ & $21(11.17)$ & $321(23.73)$ & $29(11.15)$ & $<.001$ \\
\hline Other & $932(96.88)$ & $167(88.83)$ & $1032(76.27)$ & $231(88.85)$ & \\
\hline \multicolumn{6}{|l|}{ Race } \\
\hline Nonhispanic white & $228(24.28)$ & $55(29.73)$ & $345(25.98)$ & $80(31.5)$ & .012 \\
\hline Nonhispanic African American & $61(6.5)$ & $9(4.86)$ & $107(8.06)$ & $17(6.69)$ & \\
\hline Hispanic & $86(9.16)$ & $15(8.11)$ & $82(6.17)$ & $16(6.3)$ & \\
\hline Asian & $3(0.32)$ & $1(0.54)$ & $8(0.6)$ & $1(0.39)$ & \\
\hline Other & $561(59.74)$ & $105(56.76)$ & $786(59.19)$ & $140(55.12)$ & \\
\hline \multicolumn{6}{|l|}{ Med[IQR] } \\
\hline Age (d) & $3[1,7]$ & $2[1,4]$ & $1[0,2]$ & $2[1,4]$ & $<.0001$ \\
\hline Gestational age (wk) & $39[37,40]$ & $38[36,40]$ & $39[37,40]$ & $38[37,40]$ & .9042 \\
\hline Birth weight $(\mathrm{g})$ & $3140[2640,3573]$ & $3175[2608,3515]$ & $3170[2787,3490]$ & $3025[2585,3430]$ & .0402 \\
\hline
\end{tabular}

Patients ( $=2763$ ) were identified from 24 children's hospitals across the United States (1996-2006). Values for $P$ compare demographic characteristics; numbers in parentheses show percentages of the total. $C o A$, Coarctation of the aorta; $A S$, aortic stenosis; $H L H S$, hypoplastic left heart syndrome; IAA, interruption of the aortic arch; Med IIQR], interquartile range. 
TABLE 3. Frequency of specific secondary diagnoses and chromosomal and congenital anomalies in newborns with left-sided congenital heart diseases

\begin{tabular}{|c|c|c|c|c|c|}
\hline & $\operatorname{CoA}(n=962)$ & Congenital AS $(\mathbf{n}=188)$ & HLHS $(n=1353)$ & IAA $(n=260)$ & $\boldsymbol{P}$ \\
\hline \multicolumn{6}{|l|}{ Secondary diagnoses } \\
\hline VSD & $422(43.87)$ & 45 (23.94) & $156(11.53)$ & $229(88.08)$ & $<.0001$ \\
\hline DORV & $0(0.00)$ & $0(0.00)$ & $41(3.03)$ & $9(3.46)$ & $<.001$ \\
\hline TGA & $0(0.00)$ & $0(0.00)$ & $10(0.74)$ & $4(1.54)$ & $<.001$ \\
\hline AV canal & $43(4.47)$ & $1(0.53)$ & $31(2.29)$ & $5(1.92)$ & .002 \\
\hline \multicolumn{6}{|c|}{ Chromosomal anomalies } \\
\hline Turner syndrome & $55(5.7)$ & $8(4.3)$ & $16(1.2)$ & $2(0.8)$ & $<.0001$ \\
\hline Other & $76(7.9)$ & $1(0.5)$ & $29(2.1)$ & $31(11.9)$ & \\
\hline None & $831(86.4)$ & $179(95.2)$ & $1308(96.7)$ & $227(87.3)$ & \\
\hline \multicolumn{6}{|c|}{ Congenital anomalies* } \\
\hline 0 anomalies & $707(73.49)$ & $163(86.7)$ & $1128(83.37)$ & $186(71.54)$ & $<.0001$ \\
\hline 1 anomaly & $160(16.63)$ & $14(7.45)$ & $138(10.2)$ & $50(19.23)$ & \\
\hline $2+$ anomalies & $95(9.88)$ & $11(5.85)$ & $87(6.43)$ & $24(9.23)$ & \\
\hline
\end{tabular}

An increased incidence of anomalies is associated with IAA, the left-sided congenital heart disease analyzed known to be due to a genetic defect. $P=$ differences between between secondary diagnosis or groups; numbers in parentheses show percentages of the total. CoA, Coarctation of the aorta; $A S$, aortic stenosis; $H L H S$, hypoplastic left heart syndrome; $I A A$, interruption of the aortic arch; VSD, ventricular septal defect; DORV, double-outlet right ventricle; TGA, transposition of the great arteries; $A V$ canal, atrioventricular canal. *In addition to underlying cardiac anomaly.

Of note, the majority of peak birth months for HLHS were the summer months. In contrast, congenital AS or IAA did not display the same periodicity and had weak results on Fisher's Kappa $(P=.156$ and .413 , respectively). Patients with CoA had slight evidence of seasonality with predominant birth months being April and May. Model-based monthly estimates for the rates of each diagnosis are presented in Figure 1.

We determined whether the seasonality pattern for HLHS was confounded by variables such as gender, race, birth weight, and gestational age by comparing these demographics in peak and trough months (Table 5). No statistically significant differences were found in the characteristics compared. As well, we performed a sensitivity analysis to see whether any secondary diagnosis contributed to the seasonality pattern; there was none (data not shown).

Further statistical tests of seasonality for birth month were applied to the data (Table 4). Fisher's Kappa test of the null hypothesis showed that the series is gaussian white noise, with the alternative hypothesis being that the series has a periodic component. The Barlett Kolmogorov-Smirnov test compared the normalized cumulative periodogram with the cumulative distribution function of the uniform and tested the null hypothesis that the series is white noise. With both tests, statistical significance of seasonality $(P<.0001)$ was found only with HLHS.

Finally, the $R_{\text {Autoreg }}^{2}$ value indicated moderate to strong strength of seasonality for HLHS $(=0.4575)$ compared with weak strengths for other left-sided congenital heart diseases $(<0.4)$. Furthermore, the peaks (monthly maximum) for HLHS were estimated to be in the summer (June) with troughs in the winter (January). Although small differences (between maximum and minimum values) were seen for other left-sided congenital heart diseases, the magnitude of seasonal differences per 100,000 births was greatest for HLHS.

\section{DISCUSSION}

The present study is the largest epidemiologic study of HLHS patients $(n=1353)$ reported. The population analyzed represents a relatively contemporaneous group from a diverse geographic distribution across the United States. Previously, the largest study of HLHS conducted was by Siffel, Alverson, and Correa, ${ }^{13}$ who analyzed 233 HLHS patients ( $\sim 10$ HLHS patients a year) in 5 counties of Atlanta, from 1978 to 2001.

The most significant finding of our study was the preponderant presentation of HLHS index cases in summer months during a 10-year period. This was in contrast for the overall random pattern of other left-sided congenital heart diseases. Siffel, Alverson, and Correa ${ }^{13}$ reported an absence of any seasonality for HLHS when looking at the 1978 to 2001 period overall. They noted, however, that when they limited their analysis to the 1995 to 2001 era, there was significant evidence of seasonality in HLHS. They attributed this discrepancy in part to the limitations of seasonality studies that do not use the more rigorous statistical tools such as time-series analysis, ${ }^{15,17,18}$ used in our analysis. However, the difference in era effects may have been secondary to poor survival and limited prenatal detection of HLHS infants in the 1970s and 1980s, leading to relatively smaller numbers of these cases in the earlier period. Indeed, nearly all prior epidemiologic studies of left-sided congenital heart diseases, and HLHS in particular, have been from an earlier era when HLHS survival outcomes were still less than optimal. ${ }^{4,11}$ In particular, ascertainment 

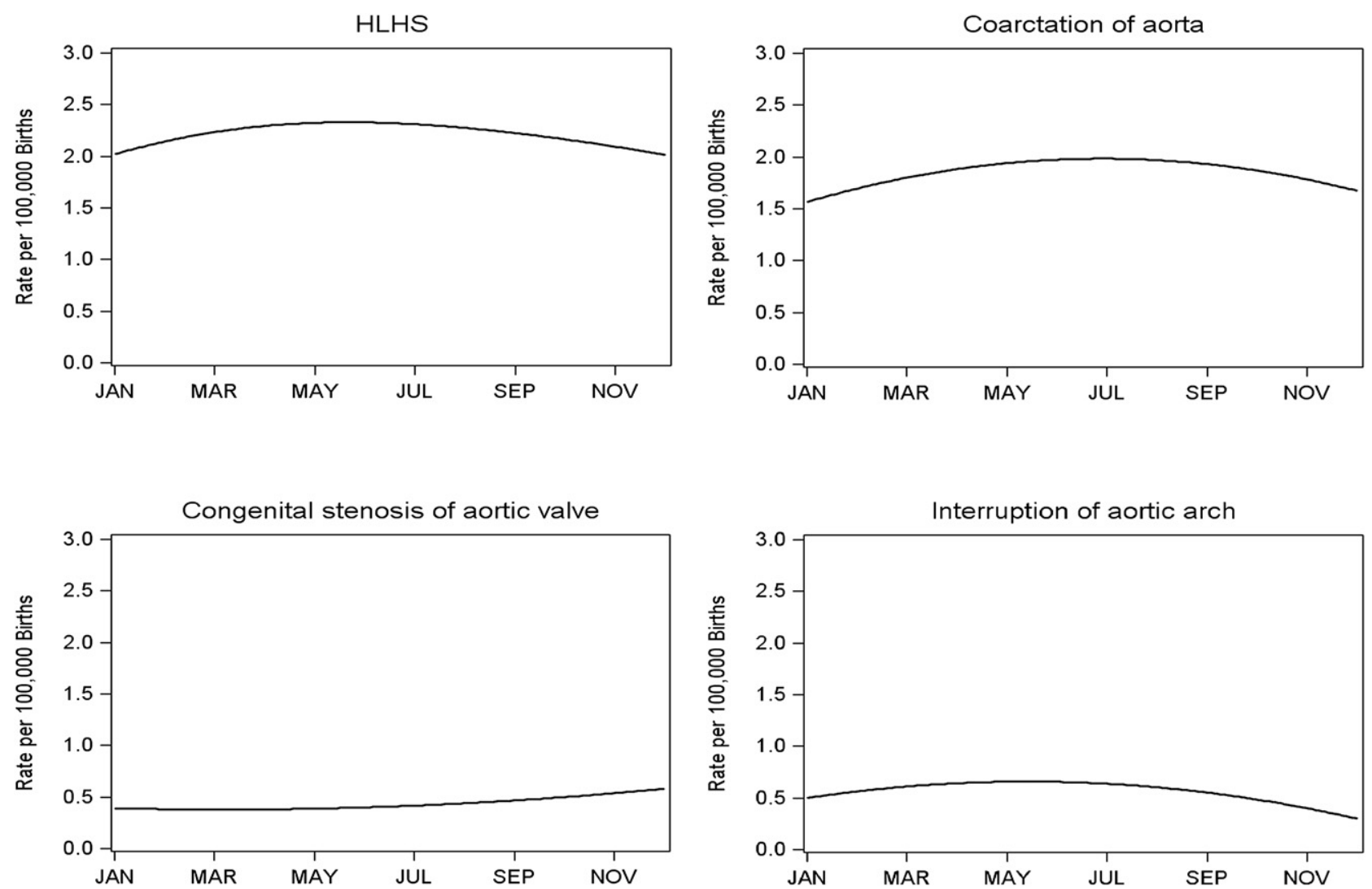

FIGURE 1. Seasonal trends in birth months (1996-2006). Model-based monthly estimates for the rates per 100,000 births for HLHS, CoA, congenital AS and IAA. The data show a seasonal trend in the occurrence of HLHS and CoA, but not in congenital AS or IAA. HLHS, Hypoplastic left heart syndrome; CoA, coarctation of the aorta; $A S$, aortic stenosis; IAA, interrupted aortic arch.

bias can be substantial when relying on autopsy reports. ${ }^{19}$ To detect the sinusoidal pattern in all seasonal diseases, one must obtain a large enough sample size to allow separation of the signal from background noise.

Our data are in concordance with overall prevalence rates for left-sided congenital heart diseases in populations from different geographic locations and time periods. ${ }^{11,12}$ Of note, the demographic characteristics, secondary diagnoses, and outcome measures of mortality were consistent with prior reports ${ }^{1,2,11,20}$ highlighting the likelihood of our study population being representative of other HLHS populations. Although single-institution reports indicate improving mortality outcomes for newborns with
HLHS, ${ }^{21}$ our mortality data are similar to those from other multi-institutional studies. ${ }^{22}$ We confirmed a greater prevalence in boys for all the left-sided congenital heart diseases analyzed except IAA. This was first demonstrated in the Baltimore-Washington Infant Study ${ }^{19}$ and subsequently by others. ${ }^{2,4,10}$ The reason for this gender predilection is unknown. Besides gender, epidemiologic studies show that certain individuals are more susceptible to left-sided congenital heart diseases than others. For example, the Baltimore-Washington Infant Study first demonstrated a higher prevalence of HLHS among whites, a finding confirmed by our study and others, including the California Birth Defects Monitoring Program ${ }^{11}$ and the Texas Birth Defects

TABLE 4. Statistical tests for seasonality of left-sided congenital heart diseases

\begin{tabular}{|c|c|c|c|c|}
\hline & $\operatorname{CoA}(n=962)$ & Congenital AS $(\mathbf{n}=188)$ & HLHS $(n=1353)$ & IAA $(\mathbf{n}=\mathbf{2 6 0})$ \\
\hline Fisher's Kappa & $5.67(.161)$ & $5.72(.156)$ & $15.86(<.001)$ & $4.69(.413)$ \\
\hline Bartlett & $0.12(.394)$ & $0.08(.821)$ & $0.30(<.001)$ & $0.12(.325)$ \\
\hline$R_{\text {Autoreg }}^{2}$ & 0.2919 & 0.1508 & 0.4575 & 0.1116 \\
\hline Monthly minimum estimate & 1.26 (Jun) & $0.23(\mathrm{Feb})$ & 1.61 (Jan) & 0.41 (Oct) \\
\hline Monthly maximum estimate & 2.51 (Apr) & 0.71 (Nov) & $2.87(\mathrm{Jul})$ & 0.83 (Oct) \\
\hline Magnitude & 1.25 & 0.48 & 1.26 & 0.420 \\
\hline
\end{tabular}

The values for Fisher's Kappa and the Bartlett Kolmogorov-Smirnov statistical tests are shown with $P$ values in parenthesis. The $R_{\text {Autoreg }}^{2}$ value for HLHS indicates moderate to strong seasonality. COA, Coarctation of the aorta; $A S$, aortic stenosis; $H L H S$, hypoplastic left heart syndrome; IAA, interruption of the aortic arch. 
TABLE 5. Comparison of HLHS patients born in the 2 highest and the 2 lowest incidence months of each year

\begin{tabular}{lccc}
\hline & $\begin{array}{c}\text { Highest } \\
\text { months N }(\%) \\
(\mathbf{n}=\mathbf{6 0 8})\end{array}$ & $\begin{array}{c}\text { Lowest } \\
\text { months N }(\%) \\
(\mathbf{n}=\mathbf{4 2 7})\end{array}$ & $\boldsymbol{P}$ \\
\hline Sex & & & \\
Female & $243(40.0)$ & $174(40.8)$ & .801 \\
Male & $365(60.0)$ & $253(59.3)$ & \\
Disposition & & & \\
Died & $109(17.9)$ & $51(11.9)$ & .009 \\
Other & $499(82.1)$ & $376(88.1)$ & \\
Race & & & \\
Nonhispanic white & $297(52.7)$ & $203(50.4)$ & .620 \\
Nonhispanic African & $58(10.3)$ & $49(12.2)$ & \\
$\quad$ American & $66(11.7)$ & $57(14.1)$ & \\
Hispanic & $6(1.1)$ & $5(1.2)$ & \\
Asian & $137(24.3)$ & $89(22.1)$ & \\
Other & $3040[2700,3420]$ & $3025[2585,3430]$ & .0408 \\
Birth weight & & & \\
$\quad$ (g): Med[IQR] & $1[0.5]$ & $1[0,4]$ & .821 \\
Age (d) Med[IQR] & $39[37,40]$ & $38[37,40]$ & .171 \\
Gestational age (d): & & & \\
Med[IQR] & & & \\
\hline There were no significant differences in the prevalence of HLHS compared with the \\
demographic parameters shown. HLHS, Hypoplastic left heart syndrome; IQR, inter- \\
quartile range.
\end{tabular}

Registry. ${ }^{4}$ These findings suggest that certain genetic makeups may influence the predisposition to left-sided congenital heart diseases.

Several etiologic factors have been implicated in the pathogenesis of left-sided congenital heart diseases such as HLHS, although a genetic mechanism has garnered the greatest interest. ${ }^{23}$ This conclusion is mainly based on heritability estimations, which can, however, have significant limitations that can be magnified if based on inclusion criteria and definition(s) of congenital heart defects (eg, is a dilated aortic root a congenital anomaly or secondary phenotype in an affected adult?). Importantly, this bias has driven the search for candidate genes to a limited set derived from primary cardiac developmental genes. Studies such as ours $^{5,24}$ suggest that alternative genetic predispositions to perhaps environmental factors may also play a part. Unexplained geographic differences have been noted for both HLHS and CoA. A follow-up study of the Baltimore-Washington Infant Study reported HLHS cases occurring as clusters within certain ZIP codes within Maryland, which were unaccounted for based on family history of cardiac malformations. ${ }^{25}$ Other reports of "clustering" of HLHS cases and evidence of association of maternal symptoms of upper respiratory infection and fever during early pregnancy, with an increase in HLHS, ${ }^{9}$ also suggest potential environmental etiologic factors that could give rise to these "clusters" of HLHS. Clearly, HLHS is a syndrome with a heterogeneic phenotypic pattern and likely heterogeneic etiologies. Other suggested etiologies for the pathogen- esis of HLHS include any restriction of inflow early in gestation, primary AS or left ventricular dysfunction leading to defective left ventricular performance, and fusion of aortic valve cusps. The mechanisms leading to these events (eg, developing aortic or mitral stenosis) have not been defined.

It is of interest that we found no evidence of seasonality among patients with congenital AS. Several case series show that fetal AS can evolve into HLHS, although there are also reports of HLHS being diagnosed in fetuses without the interval AS. It is conceivable that only certain "subsets" of congenital AS, that is, those that are precursor lesions to HLHS, follow a seasonal pattern. Our data cannot distinguish those possibilities. Interestingly, we did not find any evidence of seasonality for IAA, which is associated with chromosome 22q11 deletion in at least $50 \%$ of the patients. As well, IAA infants showed no strong evidence of gender predilection.

The importance of seasonality in the manifestation of certain diseases is gaining considerable interest. Seasonal occurrence of croup, asthma, and pneumonia has yielded information regarding their etiology, management, and prevention strategies (eg, influenza viruses or air pollution, respectively). Similarly, the strong birth prevalence of HLHS index cases during summer months would suggest that the putative environmental factor, if there is one, is most prevalent during the fall and winter months. This conclusion is based on the assumption of an 8-to 9-month gestation, inasmuch as most HLHS babies are born at or close to term. We have previously proposed a hypothesis to account for one possible environmental factor, ${ }^{6}$ although a number of other possibilities could be contemplated.

An inherent limitation of our retrospective study, which is dependent on a large database such as the PHIS, is the reliance for validity of the data as submitted by the different representative hospitals. For example, if a patient simply had a diagnosis of CoA and no reference or identification was made of a "minor" degree of AS, then the diagnosis is incorrectly classified as CoA. Ideally, the original echocardiogram(s) should be consulted for diagnostic validity. There is no reason to believe, however, that any selective diagnosis of one primary lesion versus others would have been systematically made. Missing data and coding errors are other limitations of databases such as PHIS, and although we audited all the data fields blinded to date of birth, it is possible there has been some misclassification of cases of HLHS, and possibly other lesions, using ICD-9 diagnoses. Moreover, any study of cardiovascular malformations in a population should ideally include all affected babies and pregnancies, including those ending in spontaneous abortion, termination, or stillbirth. There is evidence of a high prevalence of cardiovascular malformations in stillbirths, but reliable ascertainment is difficult to obtain. Our study, which includes only live births, could therefore miss such affected babies, and one might expect that more severe lesions (eg, HLHS) 
would be underrepresented in our data than the more minor lesions (eg, CoA or mild congenital AS) from that perspective. Unless such hidden mortality occurred selectively (ie, in certain months), our data would only be further strengthened with complete ascertainment. A further bias of our study, as noted in the Methods section, is that the database analyzed is limited to hospitals that make up the PHIS, that is, mainly tertiary care centers. Finally, a significant limitation of a study such as this is the limited ability to hold the "magnifying lens" on the diagnosis: HLHS is a syndrome in part because various anatomic subtypes can be classified as such. Therefore, we cannot assess the potential anatomic subtype(s) that make up the observed peaks. In future, perhaps other studies could elucidate this matter.

\section{CONCLUSIONS}

An analysis of left-sided congenital heart diseases from a large multi-institutional database over a 10 -year period shows significant seasonality in babies born with HLHS with peak births in summer months. Our data show an overall increase in the prevalence of both HLHS and CoA in the United States. These findings could bear significant implications for etiologic mechanisms and warrant further study.

\section{References}

1. Boneva RS, Botto LD, Moore CA, Yang Q, Correa A, Erickson JD. Mortality associated with congenital heart defects in the United States: trends and racial disparities, 1979-1997. Circulation. 2001;103:2376-81.

2. Connor JA, Arons RR, Figueroa M, Gebbie KM. Clinical outcomes and secondary diagnoses for infants born with hypoplastic left heart syndrome. Pediatrics. 2004;114:e160-5.

3. Goldberg CS, Gomez CA. Hypoplastic left heart syndrome: new developments and current controversies. Semin Neonatol. 2003;8:461-8.

4. McBride KL, Marengo L, Canfield M, Langlois P, Fixler D, Belmont JW. Epidemiology of noncomplex left ventricular outflow tract obstruction malformations (aortic valve stenosis, coarctation of the aorta, hypoplastic left heart syndrome) in Texas, 1999-2001. Birth Defects Res A Clin Mol Teratol. 2005;73:555-61.

5. Sedmera D, Cook AC, Shirali G, McQuinn TC. Current issues and perspectives in hypoplasia of the left heart. Cardiol Young. 2005;15:56-72.

6. Eghtesady P. Hypoplastic left heart syndrome: rheumatic heart disease of the fetus? Med Hypotheses. 2006;66:554-65.
7. Hinton RB Jr, Martin LJ, Tabangin ME, Mazwi ML, Cripe LH, Benson DW. Hypoplastic left heart syndrome is heritable. J Am Coll Cardiol. 2007;50: 1590-5.

8. Weismann CG, Gelb BD. The genetics of congenital heart disease: a review of recent developments. Curr Opin Cardiol. 2007;22:200-6.

9. Tikkanen J, Heinonen OP. Risk factors for hypoplastic left heart syndrome. Teratology. 1994;50:112-7.

10. Kuehl KS, Loffredo CA. Genetic and environmental influences on malformations of the cardiac outflow tract. Expert Rev Cardiovasc Ther. 2005;3:1125-30.

11. Pradat P, Francannet $C$, Harris JA, Robert E. The epidemiology of cardiovascular defects, part I: a study based on data from three large registries of congenital malformations. Pediatr Cardiol. 2003;24:195-221.

12. Fixler DE, Pastor P, Sigman E, Eifler CW. Ethnicity and socioeconomic status: impact on the diagnosis of congenital heart disease. J Am Coll Cardiol. 1993; 21:1722-6.

13. Siffel C, Alverson CJ, Correa A. Analysis of seasonal variation of birth defects in Atlanta. Birth Defects Res A Clin Mol Teratol. 2005;73:655-62.

14. Westfall JM, McGloin J. Impact of double counting and transfer bias on estimated rates and outcomes of acute myocardial infarction. Med Care. 2001;39:459-68.

15. Moineddin R, Upshur RE, Crighton E, Mamdani M. Autoregression as a means of assessing the strength of seasonality in a time series. Popul Health Metr. 2003; $1: 10$.

16. Jones KL. Smith's recognizable patterns of human malformation. 5 th ed. Philadelphia: WB Saunders; 2005.

17. Dickey D, Hasza D, Fuller W. Testing for unit roots in seasonal time series. J Am Stat Assoc. 1984;79:355-67.

18. Upshur RE, Knight K, Goel V. Time-series analysis of the relation between influenza virus and hospital admissions of the elderly in Ontario, Canada, for pneumonia, chronic lung disease, and congestive heart failure. Am J Epidemiol. 1999;149: 85-92.

19. Ferencz C, Rubin JD, McCarter RJ, Brenner JI, Neill CA, Perry LW, et al. Congenital heart disease: prevalence at livebirth. The Baltimore-Washington Infant Study. Am J Epidemiol. 1985;121:31-6.

20. McBride KL, Pignatelli R, Lewin M, Ho T, Fernbach S, Menesses A, et al. Inheritance analysis of congenital left ventricular outflow tract obstruction malformations: segregation, multiplex relative risk, and heritability. Am J Med Genet A. 2005;134:180-6.

21. Azakie T, Merklinger SL, McCrindle BW, Van Arsdell GS, Lee KJ, Benson LN, et al. Evolving strategies and improving outcomes of the modified norwood procedure: a 10-year single-institution experience. Ann Thorac Surg. 2001;72: 1349-53.

22. Gordon BM, Rodriguez S, Lee M, Chang RK. Decreasing number of deaths of infants with hypoplastic left heart syndrome. J Pediatr. 2008;153:354-8.

23. Grossfeld PD. Hypoplastic left heart syndrome: it is all in the genes. J Am Coll Cardiol. 2007;50:1596-7.

24. Van Asselt KM, Kok HS, van der Schouw YT, Peeters PH, Pearson PL, Grobbee DE. Role of genetic analyses in cardiology: part II: heritability estimation for gene searching in multifactorial diseases. Circulation. 2006;113:1136-9.

25. Kuehl KS, Loffredo CA. A cluster of hypoplastic left heart malformation in Baltimore, Maryland. Pediatr Cardiol. 2006;27:25-31. 